\title{
Dysregulation of miR-637 Is Involved in the Development of Retinopathy in Hypertension Patients and Serves a Regulatory Role in Retinol Endothelial Cell Proliferation
}

\author{
Weiguang Yang ${ }^{a}$ Manxia Su ${ }^{b}$ Yanli Yu ${ }^{b}$ Qingxin Fang ${ }^{b}$ Yusheng $M^{a}$ \\ Jihong Zhang ${ }^{c}$ \\ a Ophthalmology Department, Yidu Central Hospital of Weifang City, Weifang, China; ${ }^{b}$ Health Care Integrated \\ Geriatrics Department, Yidu Central Hospital of Weifang City, Weifang, China; 'Department of Critical Care Medicine, \\ Weifang People's Hospital, Weifang, China
}

\section{Keywords}

MiR-637 · Hypertensive retinopathy · Diagnosis · Retinal endothelial cell · STAT3

\begin{abstract}
Background: MicroRNAs play an important role in the proliferation and migration of retinal endothelial cells in patients with hypertension and hypertensive retinopathy (HR). This study aimed to investigate the clinical value of miR-637 in $\mathrm{HR}$ and its role in retinal endothelial cell proliferation and migration. Methods: A total of 126 subjects were recruited for the study, including 42 patients with hypertension (male/ female 25/17), 42 healthy individuals (male/female 20/22), and 42 cases with HR (male/female 20/22). Except SBP and $D B P$, there was no significant difference in other indexes among the three groups. Quantitative real-time PCR was used to detect the expression of miR-637. The receiver operating curve (ROC) was used for diagnosis value analysis. Logistic regression analysis was used to evaluate the relationship between miR-637 and HR. CCK-8 and Transwell were used to detect the effect of miR-637 on the proliferation and migration of human umbilical vein endothelial cells. Results: Compared with hypertensive patients, HR patients had the
\end{abstract}

Karger@karger.com www.karger.com/ore

Karger!"

BOPEN ACCESS
(C) 2022 The Author(s)

Published by S. Karger AG, Basel

This is an Open Access article licensed under the Creative Common Attribution-NonCommercial-4.0 International License (CC BY-NC) (http://www.karger.com/Services/OpenAccessLicense), applicable to the online version of the article only. Usage and distribution for commercial purposes requires written permission. lowest expression of miR-637. The area under the curve of miR- 637 detected by the ROC curve method is 0.892 , which has the ability to distinguish hypertension and HR patients. Logistic regression analysis showed that miR-637 was an independent influencing factor in HR. Cell experiment results showed that overexpression of miR-637 significantly inhibited cell proliferation and migration, while downregulation of miR637 had the opposite effect. Luciferase analysis showed that STAT3 was the target gene of miR-637. Conclusion: Our data indicate that miR-637 is a potential noninvasive marker for patients with HR. The action of miR-637 on STAT3 may inhibit the proliferation and migration of retinal endothelial cells, providing a possible target for the treatment of HR.

(c) 2022 The Author(s).

Published by S. Karger AG, Basel

\section{Introduction}

Hypertension is a main independent risk factor in cardiovascular disease and mortality worldwide [1]. One of the complications of hypertension is retinopathy, which

Weiguang Yang and Manxia Su contributed equally to this work.
Correspondence to:

Jihong Zhang, jihong@tom.com 
can not only reflect the pathological degree of systemic blood vessels and organs such as the heart, brain, and kidney but also is one of the main causes of vision loss and even blindness [2]. Endothelial cells form a thin inner layer that serves as an anatomical and functional interface between the circulating fluid in the cavity and the rest of the vessel wall. The main functions of endothelial cells include regulation of vascular tone, fluid filtration, cell recruitment, hormone transport, and hemostasis [3]. It is worth noting that, according to reports, endothelial function in patients with hypertension is impaired [4]. In addition, microvascular changes in hypertensive retinopathy (HR) are also indicators of other end-organ damage and clinical events [5].

The mass data have proven that microRNAs (miRNAs) can regulate gene expression via translation inhibition and post-transcriptional degradation [6]. The relative role of different miRNAs in vascular biology is a potential regulator of post-transcription. Therefore, miRNAs may become potential biomarkers or drug targets in vascular remodeling [7]. For example, Santulli et al. demonstrated in miRNAs and endothelial cell (DyS) function studies that miR-637 expression analysis can distinguish the white coat and non-white coat hypertension individuals [7]. A study about the upregulation of circular RNA has-circ-0037909 promotes essential hypertension mentioned that since endothelial cells are related to the pathology of target tissue damage from hypertension, Bao et al. [8] used TNF-alpha to induce the formation of EH mimics and examined changes about has-circ-0037909 and hsa-miR-637. Hence, a comprehensive understanding of the role of miRNA in endothelial dysfunction and hypertension and the molecular mechanism of miRNA action may provide novel diagnostic biomarkers and therapeutic targets for controlling hypertension-related endothelial dysfunction and other cardiovascular complications. According to the research progress, this paper mainly studies the expression level of miR-637 in patients with HR and discusses its clinical application value and its effect on retinal endothelial cell proliferation and migration.

\section{Materials and Methods}

\section{Subjects}

This study was approved by the Research Ethics Committee of Weifang People's Hospital (approval no. 2018437). Subjects were informed of the research purpose and experimental procedures before participating and signed the consent form approved by the Ethics Committee. The research method complies with the standards stipulated in the Declaration of Helsinki.
126 individuals were recruited, of which 42 cases were in the healthy group (52.24 \pm 3.89 years old, male/female 20/22), 42 cases were in the hypertension group $(52.26 \pm 4.94$ years old, male/ female 25/17), and 42 were in the HR group ( $52.95 \pm 4.71$ years old, male/female 20/22). The diagnosis of hypertension was determined in accordance with the definition of the World Health Organization, according to which hypertension is defined as the systolic blood pressure level at $>140 \mathrm{~mm} \mathrm{Hg}$ and/or diastolic blood pressure at $>90 \mathrm{~mm} \mathrm{Hg}$ [9]. Standard mercury sphygmomanometer was used to measure both systolic and diastolic blood pressure in the left arm. Blood pressure was recorded three times, and their mean values were taken. All hypertension patients were diagnosed for more than 5 years and received hypertension-related medication (such as irbesartan, carvedilol, nifedipine). Patients who had a history of coronary heart disease, smoking, diabetes, cardiovascular and cerebrovascular disease, and cancer were excluded.

\section{Clinical Samples Collection}

All participants underwent ophthalmology examinations according to the Eighth Report of the Joint National Committee. Blood samples were collected between 8:30 and 9 oclock, and after one night of fasting, to avoid differences in day-night changes. Enzyme colorimetry (Roche Diagnostics, Mannheim, Germany) was used to determine lipid profiles. Fasting blood glucose levels were measured by enzyme colorimetry (GLU, Roche Diagnostic $\mathrm{GmbH}$.

Total RNA Extraction and Quantitative Real-Time PCR Assay Total RNA was isolated from different cells and serum using TRIzol reagent according to the instructions. The isolated RNA was reverse transcribed into CDNA using PrimeScript RT Master Mix kit (Cat\# RR036A, Takara, Dalian, China). In addition, quantitative real-time PCR was performed on an ABI7300 (Applied Biosystems, Foster City, CA, USA) thermal cycler using SYBR mixture and ROX detection kit (CW2602M; CW Biotech, Beijing, China). Using a comparative CT $(2-\Delta \Delta \mathrm{Ct})$ method to detect the relative miR-637 expression, the relative gene expression of U6 was used as the internal control.

\section{Cell Culture and Transfection}

Human umbilical vein endothelial cells (HUVECs) were purchased from Bena Culture collection (Beijing, China), cultured in DMEM medium containing $10 \% \mathrm{FBS}$ and cultured in a $37^{\circ} \mathrm{C}$, $5 \% \mathrm{CO}_{2}$ constant temperature incubator. In order to regulate the expression level of miR-637, cells were transfected with miR-637 mimic, miR-637 inhibitor, or their negative control (mimic NC, inhibitor NC), which was produced by Ribo Bio (Guangzhou, China). Lipofectamine 2000 (DIR, 11668-500; Invitrogen, Carlsbad, CA, USA) was transfected according to the manufacturer's instructions.

\section{Cell Proliferation Assay}

CCK-8 method was used to detect cell proliferation. HUVECs in the logarithmic growth phase were seeded in each well of a 96 plate at $5 \times 10^{3}$. Cells were detected at $0 \mathrm{~h}, 12 \mathrm{~h}, 24 \mathrm{~h}$, and 48 h. Before testing, $10 \mu \mathrm{L}$ CCK-8 reagent (DIR, abx150974; Shanghai Beyo time, China) was added and incubated for $2 \mathrm{~h}$ in an incubator. The optical density was measured at $490 \mathrm{~nm}$ using an iMark microplate absorbance meter (ELx800; Bio-Rad Laboratories, Hercules, CA, USA). 
Table 1. Demographic, clinical, and laboratory characteristics among groups

\begin{tabular}{|c|c|c|c|c|}
\hline Parameters & Healthy & Hypertension & $\mathrm{HR}$ & $p$ value \\
\hline$n$ (male and female) & $42(20 / 22)$ & $42(25 / 17)$ & $42(20 / 22)$ & 0.415 \\
\hline Age, years & $52.24 \pm 3.89$ & $52.26 \pm 4.94$ & $52.95 \pm 4.71$ & 0.715 \\
\hline BMI, $\mathrm{kg} \mathrm{m}^{-2}$ & $24.93 \pm 1.65$ & $25.37 \pm 1.80$ & $24.88 \pm 1.64$ & 0.331 \\
\hline $\mathrm{SBP}, \mathrm{mm} \mathrm{Hg}$ & $110.19 \pm 12.97$ & $148.97 \pm 5.80$ & $154.93 \pm 5.73$ & $<0.001$ \\
\hline $\mathrm{DBP}, \mathrm{mm} \mathrm{Hg}$ & $75.53 \pm 8.42$ & $100.83 \pm 5.78$ & $110.38 \pm 8.77$ & $<0.001$ \\
\hline Fasting glucose & $87.15 \pm 5.13$ & $86.78 \pm 5.89$ & $87.25 \pm 6.03$ & 0.924 \\
\hline Total cholesterol & $169.43 \pm 12.38$ & $166.60 \pm 11.48$ & $175.42 \pm 12.61$ & 0.095 \\
\hline $\mathrm{LDL}, \mathrm{mg} \mathrm{dL}^{-1}$ & $84.50 \pm 6.17$ & $85.21 \pm 5.87$ & $84.33 \pm 6.81$ & 0.792 \\
\hline $\mathrm{HDL}, \mathrm{mg} \mathrm{dL}^{-1}$ & $49.07 \pm 5.25$ & $48.88 \pm 5.59$ & $48.81 \pm 4.82$ & 0.972 \\
\hline Triglyceride, $\mathrm{mg} \mathrm{dL}^{-1}$ & $128.07 \pm 10.06$ & $129.55 \pm 9.56$ & $125.31 \pm 9.90$ & 0.146 \\
\hline
\end{tabular}

Data are expressed as $n$ or mean \pm SD. BMI, body mass index; SBP, systolic blood pressure; DBP, diastolic blood pressure; LDL, low-density lipoprotein; HDL, high-density lipoprotein; HR, retinopathy in hypertensive patients.

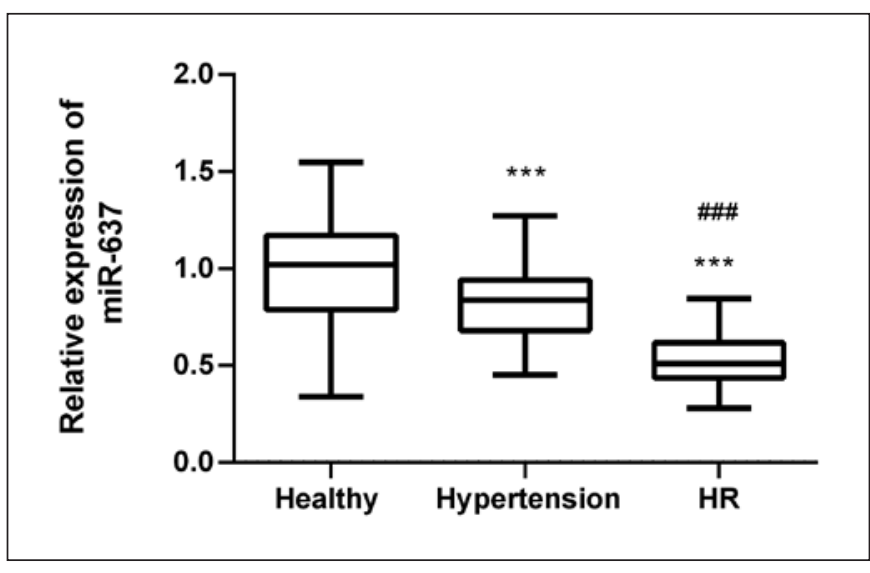

Fig. 1. Expression level of miR-637 in different groups was detected by qRT-PCR. Compared with the healthy group and hypertension patients, the expression level of miR-637 in HR patients was significantly decreased. ${ }^{* * *} p<0.001$, compared with the healthy control group; ${ }^{\# \#} p<0.001$, compared with hypertension group. qRTPCR, quantitative real-time PCR.

\section{Cell Migration Assay}

The cell migration was measured using a Transwell cell (Corning Corporation) with a pore size of $8 \mu \mathrm{m}$. Stably transfected cells $(5 \times$ $10^{4}$ cells/well) were seeded in the upper chamber of a serum-free DMEM Petri dish, and the lower chamber was filled with DMEM Petri dishes containing $10 \%$ fetal bovine serum as an attractant. At $37^{\circ} \mathrm{C}$, post-incubation cells were soaked in $0.1 \%$ crystal violet at room temperature for $20 \mathrm{~min}$ after $24 \mathrm{~h}$. Then an inverted microscope was used to observe the stained cells (Olympus Corporation Tokyo, Japan) and calculate the view from each field in the random five. The average number of migrated or invaded cells was calculated.

\section{Luciferase Reporter Assay}

The candidate target gene of miR-637 was predicted by using Target Scan Release 7.0 (http://targetscan.org/) and was

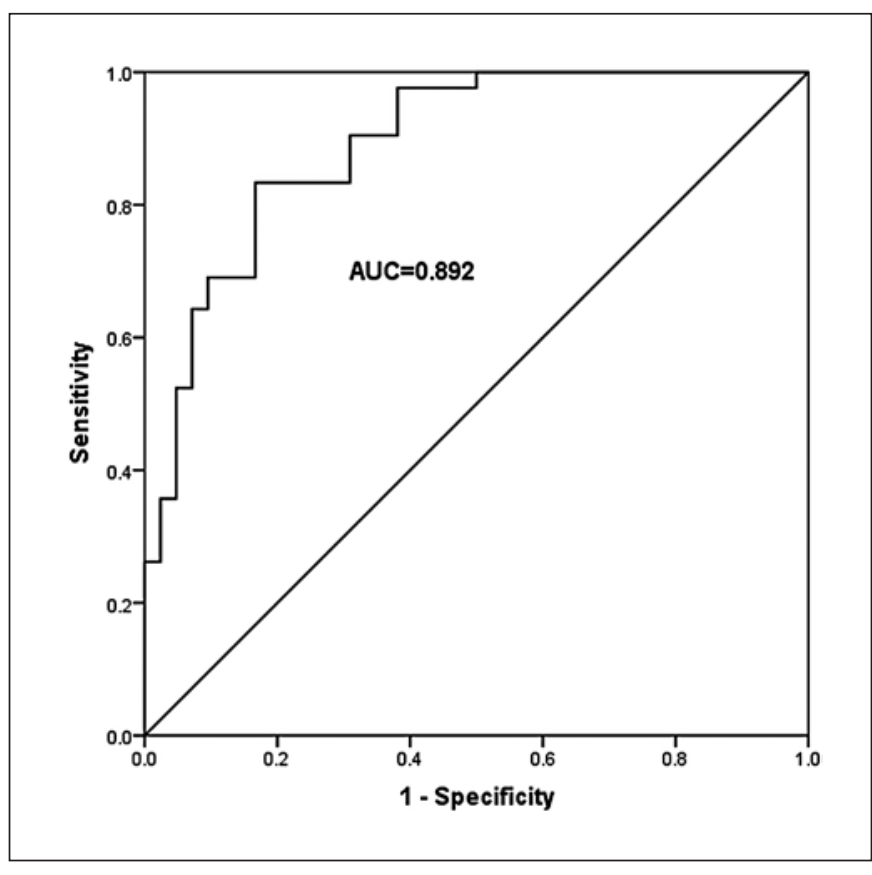

Fig. 2. ROC curve was established to assess the discriminative ability of miR-637 for identifying hypertensive patients and HR patients. The AUC was 0.892 , with a sensitivity of $83.33 \%$ and specificity of $83.33 \%$, at the cut-off value of 0.630 . AUC, area under the curve.

verified by the luciferase reporter assay. The wild type (WT) or mutant type of miR-637 in the $3^{\prime}$-UTR of STAT3 and miR-637 mimic or miR-637 inhibitor were co-transfected into HUVEC cells in each group. Lipofectamine 2000 (Invitrogen, USA) was used for cell transfection. The relative luciferase activity was measured by Dual-Luciferase Reporter System (Promega Corporation, USA) according to the instructions of the manufacturer. Renilla fluorescence activity was identified as the internal reference. 


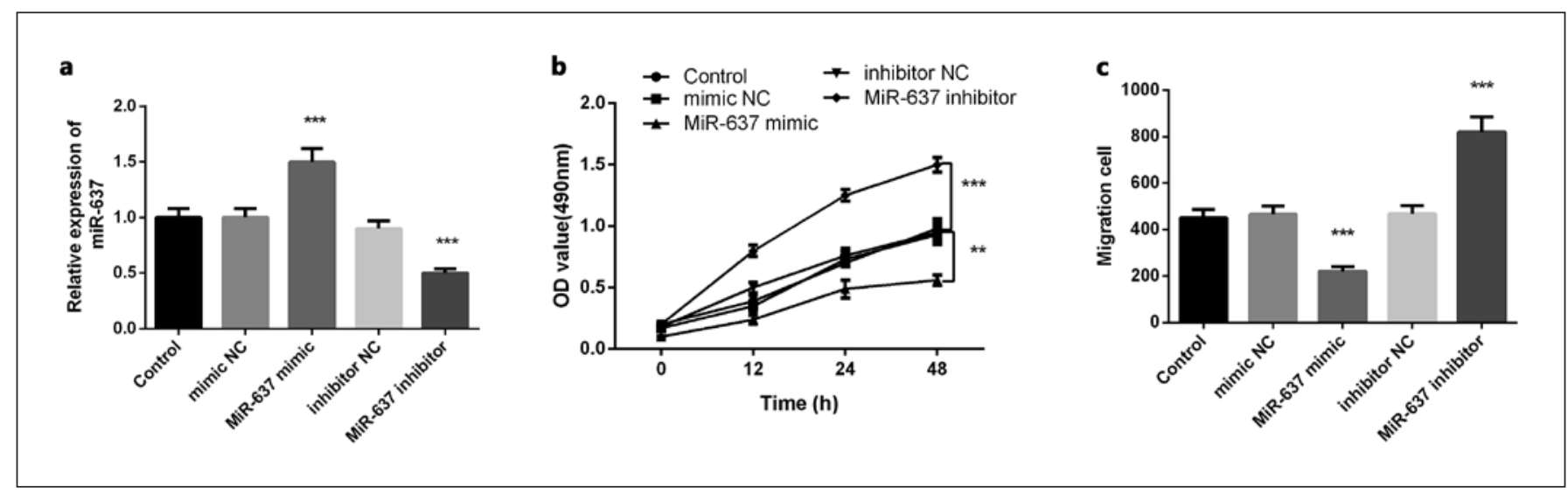

Fig. 3. Effect of miR-637 on the proliferation and migration of retinal endothelial cells. a Transfection of miR-637 mimics significantly increased the expression of miR-637, while miR-637 expression was significantly down-regulated after miR637 inhibitor transfection. b Overexpression of miR-637 significantly inhibited cell proliferation, but downregulation of miR-637 had the opposite effect. c The number of migrated cells decreased significantly after overexpressing miR-637, and the number of migrated cells increased significantly after the downregulation of miR-637. ${ }^{* *} p<0.001,{ }^{* *} p<0.01$.

Table 2. Association of different variables with the occurrence of $\mathrm{HR}$

\begin{tabular}{llll}
\hline Variables & OR & $95 \% \mathrm{Cl}$ & $p$ value \\
\hline MiR-637 & 0.290 & $0.103-0.815$ & 0.019 \\
Gender & 0.531 & $0.190-1.484$ & 0.227 \\
Age & 0.718 & $0.255-2.020$ & 0.530 \\
BMI & 1.432 & $0.529-3.877$ & 0.480 \\
SBP & 3.006 & $1.061-8.517$ & 0.038 \\
DBP & 1.941 & $0.645-5.843$ & 0.238 \\
Fasting glucose & 0.947 & $0.319-2.810$ & 0.922 \\
Total cholesterol & 0.678 & $0.242-1.903$ & 0.461 \\
LDL & 1.164 & $0.420-3.226$ & 0.770 \\
HDL & 2.578 & $0.847-7.604$ & 0.086 \\
Triglyceride & 2.162 & $0.736-6.350$ & 0.161 \\
History of anti-HT & 0.632 & $0.222-1.798$ & 0.390 \\
\hline
\end{tabular}

BMI, body mass index; SBP, systolic blood pressure; DBP, diastolic blood pressure; LDL, low-density lipoprotein; HDL, high-density lipoprotein; HR, hypertensive retinopathy; HT, hypertension.

\section{Statistical Analysis}

All data in this study were statistically analyzed using GraphPad Prism 6.0 software and SPSS21.0 software, expressed as mean and standard deviation (SD). Student's $t$ test was used to compare the statistical differences of continuous variables between the two groups, and the $\chi^{2}$ test was used to examine gender differences between the two groups. One-way ANOVA analysis was used to compare the statistical differences among different groups. Logistic regression analysis was used to assess the relationship between different variables and the occurrence of HR. The receiver operating curve (ROC) was used to evaluate the diagnostic value of miR-637. Pearson correlation analysis was used to detect the correlation between miRNA and clinical indicators. $p<0.05$ was considered statistically significant.

\section{Results}

\section{Clinical Characteristics of Different Patient Groups}

Table 1 reports the main characteristics of the study population and laboratory results. There was no difference in age, gender distribution, and BMI among the groups. Similarly, due to the results of the selection process, the metabolic parameters between the study groups were also the same. However, SBP and DBP had significant differences between the groups.

\section{Expression Levels of miR-637 in Different Patient Groups}

We first studied the serum levels of miR-637 in healthy controls, hypertension groups, and HR groups. The results of the study were shown in Figure 1. The expression level of miR-637 in the serum of patients with hypertension was significantly lower than that of the healthy control group $(p<0.05)$. In addition, this study also found that the expression level of miR-637 in patients with HR was significantly lower than that in patients with hypertension $(p<0.05)$. It was worth noting that compared with the other two groups, the expression level of HR patients was the lowest. The results indicated that miR-637 may be a key biomolecule for HR and play an important biological role in its disease progression.

\section{Diagnostic Value of miR-637 in Patients with HR}

According to the expression level of miR-637 in HR patients and a hypertension group, the ROC curve was drawn to evaluate the diagnostic value of miR-637 in HR patients. 


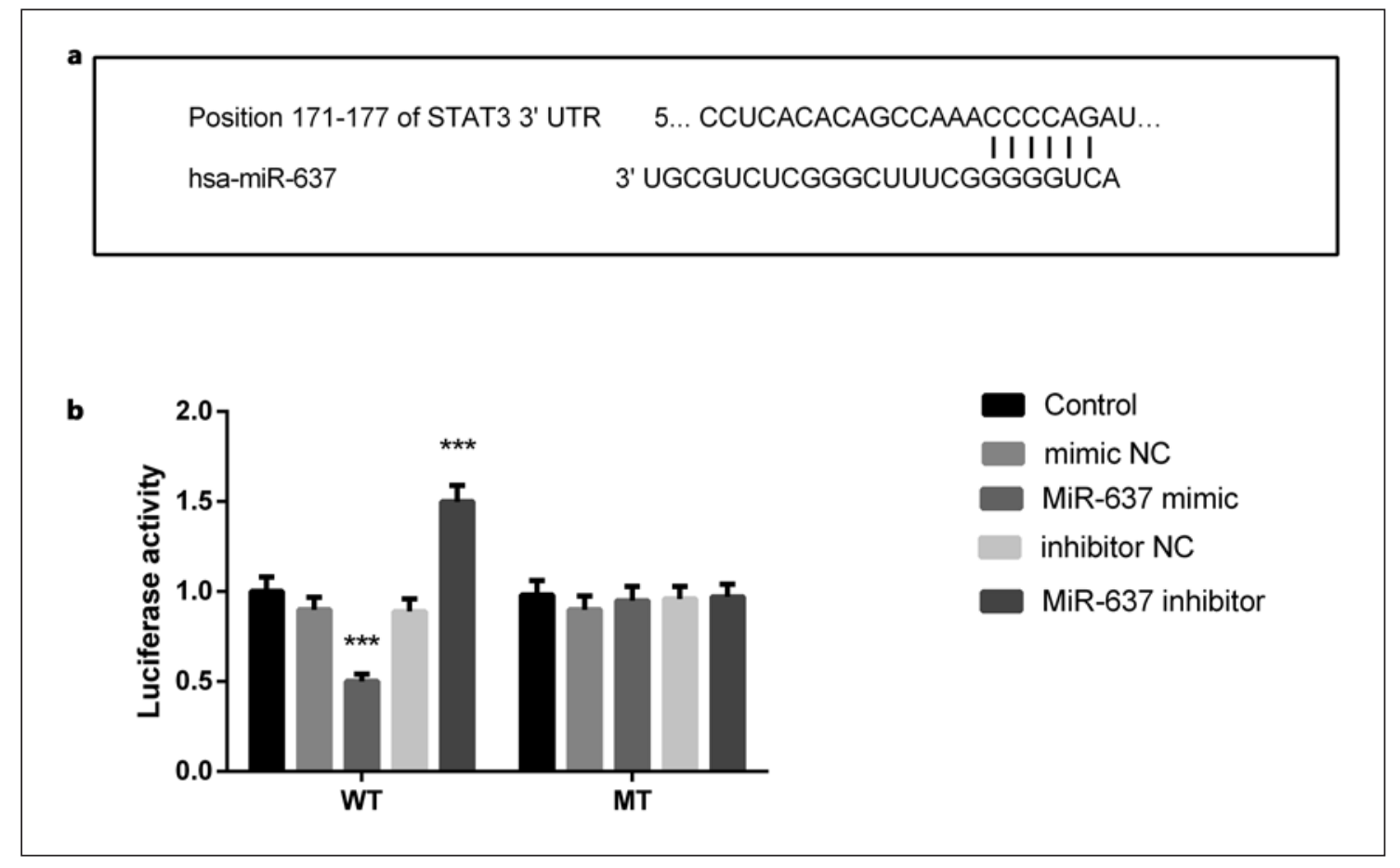

Fig. 4. STAT3 was a direct target of miR-637. a The binding site of miR-637 in STAT3. b miR-637 mimic significantly inhibited luciferase activity of WT 3'-UTR of STAT3, and miR-637 inhibitor significantly increased its luciferase activity. In addition, the luciferin activity of the mutant type group was not affected by the transfection of miR637 mimic or miR-637 inhibitor. ${ }^{* * *} p<0.001$.

As shown in Figure 2, the expression of miR-637 may be used to distinguish patients with hypertension and patients with HR. The area under the curve was 0.892 , the cut-off value was 0.630 , and the sensitivity and specificity were $83.3 \%$ and $83.3 \%$, respectively. The results of this study confirmed that miR-637 had certain diagnostic value in distinguishing hypertensive patients from HR patients.

\section{Correlation between miR-637 Expression and the}

Incidence of $H R$

Table 2 uses a logistic regression analysis to evaluate the relationship between miR- 637 and HR. As can be seen from Table 2, low level of miR-637 $(\mathrm{OR}=0.304,95 \% \mathrm{CI}=$ $0.109-0.9845, p=0.022)$ and high level of SBP (OR = $3.018,95 \% \mathrm{CI}=1.069-0.8520, p=0.037)$ were independent factors for the occurrence of HR in hypertensive patients. In summary, miR-637 was a good indicator for evaluating HR in hypertensive patients.

\section{Effects of miR-637 on the Proliferation and Migration} of Retinal Endothelial Cells

The abnormal behavior of retinal endothelial cells has been reported to play an important role in HR. In this study, we transfected miR-637 mimics or inhibitors into HUVECs to regulate the expression of miR-637. Figure 3 shows the effect of miR-637 on HUVEC proliferation and migration. As can be seen from Figure 3a, the transfection of miR-637 mimic/inhibitor had a significant effect on the expression of miR-637 ( $p<0.01)$, transfection of miR-637 mimic significantly increased the expression of miR-637, and transfection of miR-637 inhibitor played the opposite effect. As can be seen from Figure $3 \mathrm{~b}$, the results showed that overexpression of miR-637 can significantly inhibit cell proliferation, while downregulation of miR-637 had the opposite effect ( $p<$ 0.01 ). As can be seen from Figure $3 c$, the effect of the miR637 expression on cell migration was detected by the Transwell experiment. The results showed that overexpression of miR-637 can significantly reduce the number of cell migration, and downregulation of miR-637 can increase the number of cell migration $(p<0.001)$. In summary, miR-637 inhibits cell proliferation and migration in HUVECs.

\section{MiR-637 Directly Targets STAT3 in Retinal Endothelial Cells}

As is widely known, miRNAs exert their function through suppressing the expression of their target gene(s). 
According to the TargetScan analysis results, the binding site of miR-637 in STAT3 was shown in Figure 4a. Luciferase reporter assay results showed that miR-637 mimic significantly inhibited luciferase activity of WT $3^{\prime}$-UTR of STAT3 $(p<0.01)$, and miR-637 inhibitor significantly increased its luciferase activity (shown in Fig. 4b). In addition, the luciferin activity of the mutant type group was not affected by the transfection of miR-637 mimic or miR-637 inhibitor (shown in Fig. 4b).

\section{Discussion}

As previous studies described, many diseases can cause changes in retinal signals, such as hypertension, diabetes, metabolic syndrome, and heart disease [10]. $\mathrm{HR}$ is an important complication and is the main site of target organ damage caused by hypertension. At the same time, knowledge of HR is also important in the cardiovascular risk stratification of hypertensive individuals [11]. As we all know, when the blood pressure exceeds the critical limit, the automatic regulation of retinal circulation will fail [12]. Thus, determining clinical characteristics that can be used to predict the development and progression of retinopathy is essential for hypertensive patients. For example, a study mentioned that retinal vascular tortuosity and arterial narrowing due to HR and arteriolar sclerosis were noted in both eyes [13]. The specific miRNA can destroy the blood vessel-retinal barrier and selectively secrete it into the circulatory system through small membrane bubbles (such as exosomes) [14]. Our results showed that the expression level of miR-637 was highest in the healthy group without hypertension and lowest in the HR patient. Yin et al. [15] confirmed that miR-637 expression is reduced in hypertensive subjects. This is consistent with the results reported in the literature. In the meantime, we speculate that miR-637 may have a potential role in the development of the HR population.

Previous studies also confirmed that specific miRNA expression patterns and levels can reveal physiological and pathological changes and related microvascular and macrovascular complications in patients with HR and are considered to be a valuable novel biomarker [16]. For example, in a study about the role of miRNA in endothelial dysfunction and hypertension, it was reported that miR-637 targets other inflammatory pathways in order to decrease the C-reactive protein (CRP) levels in other pathological situations such as hypertension [17]. In another study, the downregulation of the miR-637, which regulates the CDK6 expression from lung smooth muscle cells, increases the risk of hypoxiainduced pulmonary hypertension [18]. In our study, the ROC curve was used to assess whether serum miR637 was capable of identifying HR patients from hypertensive cases. The results showed that miR-637 had a high diagnostic capacity between the two groups. Logistic regression analysis showed that miR-637 was an independent influencing factor for the occurrence of HR in hypertensive patients. Therefore, it may be used as a biomarker for HR patients. In addition, studies have confirmed that hypertension can cause changes in miRNAs such as miR21 , miR-122, and miR-637 [19]. This is consistent with the results of this study.

Endothelial cell dysfunction is the main feature of retinal neovascular disease, which is the main cause of blindness in developed countries [20]. Considering the crucial role of endothelial cell dysfunction in the development of HR, the dysregulation of miR-637 in HR patients, we assumed that miR-637 may regulate the proliferation and migration of retinal endothelial cells during the occurrence of HR. Cell experiments confirmed the effect of miR-637 on the proliferation and migration of HUVEC cells, suggesting that miR-637 may be involved in HR through regulating the proliferation and migration of HUVEC cells.

Signal transducer and activator of transcription protein-3 (STAT3) is a transcription factor involved in many biological processes, especially the process of cell survival and proliferation [21]. It was originally isolated from cytokine-induced cell culture, and it was later found in many normal and pathological tissues [22]. For example, it was mentioned in a study that the oxygen-induced retinopathy rat model showed increased neovascular retinal blood vessels with increased STAT3 activity [23]. Last but not least, studies have confirmed that the proliferation of endothelial cells induced by VEGF can be inhibited by STAT3 knockdown in HUVEC and human retinal microvascular endothelial cells [24]. This is consistent with our findings that STAT3 was the target gene of miR-637. Therefore, we speculated that miR-637 may regulate the proliferation of retinal endothelial cells via targeting STAT3. However, our research also has some limitations. The research population is relatively small, and a larger group is needed to verify the current results. In addition, the specific mechanism of the role of miR-637 in HR function needs further study.

In summary, our research suggests that miR-637 was less expressed in HR patients. MiR-637 is a potential marker for HR patients. miR-637 may participate in the development 
of HR patients by acting on the target gene STAT3 to inhibit the proliferation and migration of retinal endothelial cells.

\section{Statement of Ethics}

This study was approved by the Research Ethics Committee of Weifang People's Hospital (approval no. 2018437). The research method complies with the standards stipulated in the Declaration of Helsinki. Subjects were informed of the research purpose and experimental procedures before participating and signed the consent form approved by the Ethics Committee.

\section{Conflict of Interest Statement}

The authors declare that they have no competing interests.

\section{Funding Sources}

There was no funding for this study.

\section{Author Contributions}

All authors participated in the design, interpretation of the studies, analysis of the data, and review of the manuscript. Weiguang Yang, Manxia Su, Yanli Yu, and Qingxin Fang conducted the experiments. Yusheng Ma supplied critical reagents. Weiguang Yang and Manxia Su wrote the manuscript and Jihong Zhang revised it critically for intellectual content. All authors read and approved the final manuscript.

\section{Data Availability Statement}

The corresponding authors may provide data and materials.

\section{References}

1 Salkic S, Ljuca F, Batic-Mujanovic O, Brkic S, Mesic D, Mustafic S. The frequency of hypertension crises in the emergency medical service department in tuzla. Med Arch. 2013;67:393-6.

2 Samanta D, Prabhakar NR, Semenza GL. Systems biology of oxygen homeostasis. Wiley Interdiscip Rev Syst Biol Med. 2017;9(4):e1382.

3 Santulli G, Ciccarelli M, Palumbo G, Campanile A, Galasso G, Ziaco B, et al. In vivo properties of the proangiogenic peptide QK. J Transl Med. 2009;7(1):41.

4 Ward NC, Croft KD, Hodgson J, Rich L, Beilin LJ, Puddey IB. Brachial artery vasomotor function is inversely associated with 24 -h ambulatory blood pressure. J Hypertens. 2004; 22(5):967-72.

5 Bhargava M, Ikram MK, Wong TY. How does hypertension affect your eyes? J Hum Hypertens. 2012;26(2):71-83.

6 Langevin S, Kuhnell D, Parry T, Biesiada J, Huang S, Wise-Draper T, et al. Comprehensive microRNA-sequencing of exosomes derived from head and neck carcinoma cells in vitro reveals common secretion profiles and potential utility as salivary biomarkers. Oncotarget. 2017;8(47):82459-74.

7 Santulli G. MicroRNAs and endothelial (dys) function. J Cell Physiol. 2016;231(8):1638-44.

8 Bao X, He X, Zheng S, Sun J, Luo Y, Tan R, et al. Up-regulation of circular RNA hsa circ_0037909 promotes essential hypertension. J Clin Lab Anal. 2019;33(4):e22853.

9 Chobanian AV, Bakris GL, Black HR, Cushman WC, Green LA, Izzo JL Jr, et al. The seventh report of the Joint national committee on prevention, detection, evaluation, and treatment of high blood pressure: the JNC 7 report. JAMA. 2003;289(19):2560-72.
10 Wong TY, McIntosh R. Hypertensive retinopathy signs as risk indicators of cardiovascular morbidity and mortality. Br Med Bull. 2005; 73-74(1):57-70.

11 Chatterjee S, Chattopadhya S, Hope-Ross M, Lip PL. Hypertension and the eye: changing perspectives. J Hum Hypertens. 2002;16(10): 667-75.

12 Youssef G, Nagy S, El-Gengehe A, Abdel Aal A, Hamid MA. Masked uncontrolled hypertension: prevalence and predictors. Egypt Heart J. 2018;70:369-73.

13 Moradian S, Soheilian M. Periretinal hemorrhage due to retinal arterial macroaneurysm: the role of ICG angiography in solving a diagnostic dilemma. J Ophthalmic Vis Res. 2009; $4(2): 125-6$

14 V La Marca, Fierabracci A. Insights into the diagnostic potential of extracellular vesicles and their miRNA signature from liquid biopsy as early biomarkers of diabetic micro/macrovascular complications. Int J Mol Sci. 2017; 18(9):1974.

15 Yin L, Yao J, Deng G, Wang X, Cai W, Shen J. Identification of candidate lncRNAs and circRNAs regulating WNT3/ $\beta$-catenin signaling in essential hypertension. Aging. 2020;12(9): 8261-88.

16 Liu HN, Cao NJ, Li X, Qian W, Chen XL. Serum microRNA-211 as a biomarker for diabetic retinopathy via modulating Sirtuin 1 . Biochem Biophys Res Commun. 2018;505(4):1236-43.

17 Nemecz M, Alexandru N, Tanko G, Georges$\mathrm{cu}$ A. Role of MicroRNA in endothelial dysfunction and hypertension. Curr Hypertens Rep. 2016;18(12):87.

18 Sang HY, Jin YL, Zhang WQ, Chen LB. Downregulation of microRNA-637 increases risk of hypoxia-induced pulmonary hypertension by modulating expression of cyclin dependent kinase 6 (CDK6) in pulmonary smooth muscle cells. Med Sci Monit. 2016;22: 4066-72.

19 Wang J, Yan Y, Song D, Liu B. Reduced plasma miR-146a is a predictor of poor coronary collateral circulation in patients with coronary artery disease. Biomed Res Int. 2016; 2016:4285942.

20 Zhu M, Liu X, Wang Y, Chen L, Wang L, Qin $\mathrm{X}$, et al. YAP via interacting with STAT3 regulates VEGF-induced angiogenesis in human retinal microvascular endothelial cells. Exp Cell Res. 2018;373(1-2):155-63.

21 Kumar R, Singh NK, Rao GN. Proline-rich tyrosine kinase 2 via enhancing signal transducer and activator of transcription 3 -dependent cJun expression mediates retinal neovascularization. Sci Rep. 2016;6(1): 26480.

22 Takeda K, Noguchi K, Shi W, Tanaka T, Matsumoto M, Yoshida N, et al. Targeted disruption of the mouse Stat 3 gene leads to early embryonic lethality. Proc Natl Acad Sci U S A. 1997;94(8):3801-4.

23 Planas AM, Soriano MA, Berruezo M, Justicia C, Estrada A, Pitarch S, et al. Induction of Stat3, a signal transducer and transcription factor, in reactive microglia following transient focal cerebral ischaemia. Eur J Neurosci. 1996;8(12):2612-8.

24 Ye EA, Steinle JJ. miR-146a suppresses STAT3/VEGF pathways and reduces apoptosis through IL-6 signaling in primary human retinal microvascular endothelial cells in high glucose conditions. Vis Res. 2017; 139:15-22. 